\title{
Mathematical Methods
}

\author{
in \\ Medicine
}

\section{Richard Bellman}

Professor of Mathematics, Electrical Engineering and Medicine University of Southern California

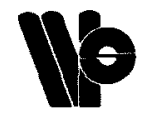

World Scientific 
World Scientific Publishing Co Pte Ltd

P O Box 128

Farrer Road

Singapore 9128

Copyright $(\odot 1983$ by World Scientific Publishing Co Pte Ltd. All rights reserved. This book, or parts thereof, may not be reproduced in any form or by any means, electronic or mechanical, including photocopying, recording or any information storage and retrieval system now known or to be invented, without written permission from the publisher.

\author{
ISBN $9971-950-20-0$ \\ 9971-950-21-9 pbk
}

Printed in Singapore by Richard Clay (S. E. Asia) Pte. Ltd. 
To

Bill Hitselberger

whose skill, courage and determination made this volume possible. 
This page is intentionally left blank

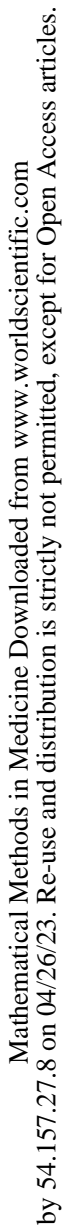




\section{PREFACE}

The purpose of this book is to show how mathematical problems arise in medicine. Our theme is the administration of drugs.

An increasing number of mathematicians, engineers and physicists want to work in the fields of physiology and medicine. The motivations are varied: idealism and a desire to be relevant; recognition of the fact that the areas are essentially wide open with large numbers of important and challenging problems; appreciation of the job opportunities for the students and themselves; a clear view of where present and future government funding will be focused.

The kinds of questions that these individuals most often ask are:

1. Where are the mathematical problems?

2. How do we learn enough physiology and medicine to fruitfully apply our mathematical and scientific skills?

3. How does one go about working with physiologists and medical doctors?

This volume is an attempt to respond to these questions, based upon about fifteen years of effort at the RAND Corporation and the University of Southern California primarily in the areas of chemotherapy and pharmacokinetics, nuclear medicine and psychotherapy. In each of these areas I was fortunate in having access to experts in their domains who were interested in the mathematical approach.

We have tried to show first by a simple example how scientific 
questions arise in natural and immediate ways. Then we wish to indicate the kinds of mathematical problems that are posed by this medical motivation, some of the analytic problems that arise in this fashion as well as methods which can be systematically employed, and, most important computational approaches that may be invoked.

Throughout we pursue a simple stubborn theme: How can we obtain numerical answers to numerical questions? More generally, what are feasible procedures for patient care?

We uniformly take the view that the role of medicine vis-a-vis physiology is completely analogous to that of engineering towards science. Each has a crucial need for the other, with the vital difference that medicine and engineering require answers now. This simple observation creates vast new domains of mathematics. Let us emphasize that our primary objective is to point out these domains.

In Chapter I, we describe one- and two-compartment models. Here, the quantities of interest can easily be obtained explicitly. In Chapter II, we consider the multi-compartment case. In Chapter III, we describe how various questions in observation and testing lead naturally to multi-point boundary value problems. Also, many problems of classical type arise naturally. In Chapter IV, we show that the compartmental model has many desirable features. Here, it is very convenient to use matrix theory.

Realistic models of biomedical phenomena often need the digital computer. In Chapter $V$, we describe some of the properties of the digital computers which we will employ. In Chapter VI, we give some typical algorithms to illustrate the type of problem that arises.

Next, we turn to the "side effect" problem. Here, we want to maintain a therapeutic level in some compartments while avoiding too high a concentration in other compartments. These questions lead naturally to various problems in the theory of control. In Chapter VII and Chapter VIII, we show how these problems can be treated by the methods of the calculus of variations. In Chapter VII, we do not 
consider clinical constraints. In Chapter VIII, we consider these constraints dictated by clinical considerations and show that they lead to considerably analytic difficulties. These clinical constraints motivate an entirely new approach to these problems. We show that dynamic programming furnishes a quick, simple and easy computational approach. The fundamentals of the theory are discussed in Chapter IX, and various operational considerations are discussed in Chapter $x$.

In Chapter XI, we show that the same methods of dynamic programming can handle many cases where uncertainty is present.

In Chapters XII and XIII, we study some questions connected with radiation. In Chapter XII, we study detection processes using radiation. The original process study, a scanning process, has been superseded by more efficient methods. However, the mathematical method developed can be applied to other medical problems, to many scientific problems, and to quality control. Hence, we keep the original medical problem to show the motivation.

In Chapter XIII, we turn to radiotherapy. This is again the side effect problem, because the radiation that destroys the diseased tissue affects the healthy tissue. Methods developed for radiative transfer can be applied here.

It will be clear how much remains to be done, and consequently, how many opportunities exist. The human system will influence the future of mathematics the way the physical systems influenced the development. 


\section{This page is intentionally left blank}




\section{CONTENTS}

PREFACE

CHAPTER I: ONE- AND TWO-COMPARTMENT MODELS

1. Introduction

2. Compartment Models

3. One-Compartment Model

4. Injections

5. Discrete Model

6. Interconnections

7. Advantages and Disadvantages

8. Two-Compartment Model

9. Analytic Solution

10. Reduction to Second-Order Linear Differential Equations

11. Discussion

12. Model-Making

13. Discussion

14. Successive Approximations

Pent Approximation

16. Perturbation Procedures 19

17. Small Rate Constant. 19

Bibliography and Comments $\quad 20$

CHAPTER II: MATRICES AND MULTI-COMPARTMENT MODELS

1. Introduction

22

2. Multi-Compartment Model

3. Discussion

4. Vectors and Matrices

5. The Exponential Matrix

7. Special Structures 28

8. Analytic and Computational Results 29

9. Conversion of Linear Systems and Constant Coefficients 29

10. Variable Coefficients 30

11. Characteristic Roots and Characteristic Vectors 31

12. Particular Integrals 31

13. Perturbation Equations 32

Bibliography and Comments 33 
CHAPTER III: OBSERVATION AND TESTING

1. Introduction 34

2. Unobservables 35

3. Necessary and Sufficient Conditions 36

4. Time Averages 37

5. Discussion 38

6. Design of Experiments 38

7. Identification 39

Bibliography and Comments 39

CHAPTER IV: PHARMACOKINETIC PROPERTIES

1. Introduction

40

2. Non-Negativity - I 41

3. Non-Negativity - II 42

4. Non-Negativity - III 42

5. Discussion 43

6. Positivity of Concentration 44

7. Conservation 44

8. Terminal Condition 45

9. Degrees of Freedom 45

10. Attainable States 46

11. Equilibrium 46

12. Mixing and Equilibrium 47

13. Mathematical Languages 48

14. M-Matrices 49

15. Adjoint Matrix 49

16. Approach to Equilibrium 50

17. Determination of Equilibrium Concentrations 51

18. Time-Dependence 52

19. Nonlinearity 52

20. Further Directions of Research 53

Bibliography and Comments 53

CHAPTER V: THE SORCERER'S APPRENTICE: THE DIGITAL COMPUTER

1. Introduction

2. Computer Revolutions 56

3. Computers 57

4. Digital Computers 58

5. Storing and Retrieving Numbers 58

6. Storage 60

7. Memory and Rapid Access Storage 61

8. Arithmetic 62

9. Wrong Arithmetic Rapidly Done 63

10. Instructions 65

11. Algorithm 68

12. Algorithm for Addition 68

13. Logical Operations 69 
14. Sorting

15. Arithmetic Solution

16. Parallel Processing

CHAPTER VI: NUMERICAL ALGORITHMS

1. Introduction

2. Linear Algebraic Systems 73

3. Operational Aspects 75

4. Discussion 76

5. Gaussian Elimination $\quad 77$

6 . Computation of the Inverse 78

7. Evaluation of Determinants 78

8. Selective Computation $\quad 79$

9. 111-Conditioned Systems 81

10. Additional Information 81

11. Square Roots 82

12. Newton-Raphson 83

13. Example 84

14. Discussion $\quad 85$

15. Some Matrix Recurrence Relations 86

16. Search Techniques 87

17. Discussion 88

18. Linear Differential Equations 89

19. Difference Methods 90

20. Validity 91

21. Stability Theory 92

22. Sources of Error 93

23. Selective Calculation 94

24. Improved Algorithm 96

25. Updating 97

26. Linear Equations with Variable Coefficients 98

27. Second Order Differential Equations 99

28. WKB Approximation 110

29. Nonlinear Equations 111

30. Stiff Equations. 111

31. Conclusion 114

Bibliography and Comments $\quad 114$

CHAPTER VII: OPTIMAL DOSAGE AND CONTROL THEORY

1. Introduction

2. A Side Effect Problem 116

3. Control Process 118

4. Actions and Effects 118

5. Evaluation of Outcomes 119

6. Discussion 120

7. Quadratic Norm 121

8. Geometric Interpretation 122 
9. Choice of Initial Concentrations 123

10. Numerical Solutions 124

11. Multi-Compartment Case 125

12. Operational Aspects 127

13. Search Techniques 128

14. Continuous Infusion 129

15. Scalar Case 129

16. The Euler-Lagrange Equation 130

17. More General Scalar Case 133

18. Minimum Property 134

19. Multi-Dimensional Case 135

20. Computational Aspects 136

21. Alternate Approach 137

22. More General Multi-Dimensional Case 138

23. Rayleigh-Ritz Method 138

24. Feasible vs. Optimal 140

25. Constraints 140

26. Inner Products 142

27. Integral Case 143

28. Nonlinear Kinetics 143

Bibliography and Comments 144

CHAPTER VIII: CLINICAL TREATMENT AND CONSTRAINTS

1. Introduction : : 145

2. A Particular Problem 146

3. Constrained Variation 146

4. Convex Variation 148

5. Neyman-Pearson Lemma 150

6. Another Approach - Convexity 153

7. The Solution $\quad 155$

8. Another Particular Problem 156

9. The Functional $\max |1-u| \quad 157$

10. Discussion 158

Bibliography and Comments $\quad 159$

CHAPTER IX: DECISION PROCESSES AND DYNAMIC PROGRAMMING

1. Introduction

2. Control Process as a Decision Process 161

3. Policy

4. Principle of Optimality

5. Discrete Models

6. One-Compartment: Formulation 163

7. Conventional Treatment 164

8. Dynamic Programing 164

9. Analytic Solution 165

10. Computational Solution 167

$\begin{array}{ll}\text { 11. Analytic Solution } & 168\end{array}$ 
12. Asymptotic Behavior 169

13. Constraints 170

14. Generalizations 171

15. Changeable Rate Constants 172

16. Continuous Models 172

17. The Analytic Formalism 174

18. Simplification 176

19. Repeated Dosage 177

20. Formulation 178

21. Time-Dependence 179

22. Threshold Effects 181

23. Multi-Compartment Models 182

24. The Riccati Equation 182

25. Invariant Imbedding 183

26. Discussion 183

Bibliography and Comments 183

CHAPTER $X:$ ANAL $¥ T I C$ AND COMPUTATIONAL TECHNIQUES

1. Introduction

184

2. One-Compartment Model 185

3. Functional Equation 185

4. Computational Aspects 186

5. Computational Procedure - Stage $1 \quad 187$

6. Discussion 188

7. Computational Procedure - Stage N 188

8. Space and Time 189

9. Additional Dosage Constraint 189

10. Two-Compartment Case 190

11. Discussion 191

12. Search Processes 192

13. Storage and Retrieval of Data-Polynomial Approximation 192

14. Splines 193

15. Stability 194

16. Multi-Compartment Models 195

17. Successive Approximations 195

18. Lagrange Multipliers 195

19. Discussion 195

$\begin{array}{ll}\text { Bibliography and Comments } & 196\end{array}$

CHAPTER XI: UNCERTAINTY

1. Introduction 197

2. Different Types of Uncertainty 197

3. Stochastic Cause and Effect 198

4. Expected Side Effect 199

5. Discrete Stochastic Control Processes 199

6. Functional Equations 200

7. Fuzzy Systems 201

8. Markov Processes 201 
9. Continuous Markov Processes 202

10. Discussion 203

11. Markovian Decision Processes 203

12. Introducing a New Drug 204

13. Mathematical Model 205

14. Assumptions 206

15. Learning 207

16. Formulation as a Multistage Decjsion Process 208

17. Expected Gain 209

18. Value of Information 209

19. Further Assumptions $\quad 210$

20. Functional Equation 210

21. Discussion 212

$\begin{array}{ll}\text { Bibliography and Comments } & 212\end{array}$

CHAPTER XII: TUMOR DETECTION AND SCAN-RESCAN PROCESSES

1. Introduction 214

2. Formulation $\quad 215$

3. Minimization 217

$\begin{array}{ll}\text { 4. Hierarchies } & 218\end{array}$

$\begin{array}{ll}\text { Bibliography and Comments } & 218\end{array}$

CHAPTER XIII: RADIOTHERAPY AND RADIATIVE TRANSFER

1. Introduction 220

2. Physical Processes 221

3. Recurrence Relations 224

4. Scattering and Transmission Functions of Finite Order 225 $\begin{array}{ll}\text { Appendix } & 242\end{array}$

$\begin{array}{ll}\text { Bibliography and Comments } & 247\end{array}$

$\begin{array}{lr}\text { SUBJECT INDEX } & 249\end{array}$

$\begin{array}{ll}\text { NAME INDEX } & 251\end{array}$ 